\title{
Lichen Sclerosus et Atrophicus with Cutaneous Distribution Simulating Lichen Planus
}

\author{
M. Gómez Vázquez ${ }^{\mathrm{a}}$ R. Navarra ${ }^{\mathrm{a}} \quad$ M.T. Martin-Urda ${ }^{\mathrm{a}}$ \\ $\begin{array}{lll}\text { C. Abellaneda } & \text { A. Quer } \\ & \end{array}$

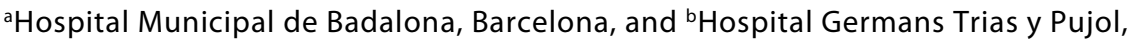 \\ Barcelona, Spain
}

\section{Key Words}

Lichen sclerosus · Lichen planus · Skin distribution

\begin{abstract}
Lichen sclerosus (LS) et atrophicus is a disease of unknown etiology, although hereditary, endocrine, and autoimmune factors are known to be involved. While the anal and genital regions are predominantly affected, only $2.5 \%$ of patients present with extragenital lesions, particularly of the trunk, neck, and upper limbs. The possible relationship between lichen sclerosus et atrophicus and both lichen planus (LP) and localized scleroderma (morphea) has not been clearly established, although in a number of cases, several of these conditions have been found simultaneously. We report the case of a 31year-old woman with LS lesions affecting the neck, upper back, wrist and dorsum of the feet. The unusual character of this presentation is pointed out, along with its clinical similarity to LP.
\end{abstract}

\section{Case Report}

Lichen planus (LP) and lichen sclerosus (LS) share similar pathological and clinical features. There have been a few reported cases in the literature of the coexistence of LP and LS [1-3]. We report a case of LS with cutaneous distribution simulating LP.

A 31-year-old woman presented with a 1-year history of a severe pruritic eruption that initially developed on the dorsum of the feet and posteriorly also appeared on the neck, upper back and wrists (fig. 1, fig. 2). Exploration revealed small, centrally depressed, polygonal macular lesions on the wrists and dorsum of the feet. The distribution of the lesions was bilateral and symmetrical. Moreover, multiple atrophic papules of pearly coloration, bright and 5-8 $\mathrm{mm}$ in size on the neck and upper back were present. There were no lesions affecting the oral mucosa, genitals, or rest of the body. Physical examination was otherwise normal. Laboratory studies including hemogram, biochemistry, antibodies against thyroid and antinuclear antibodies, and borrelia, hepatitis B and C virus serology were within normal ranges or were negative. 


\begin{tabular}{l|l|l|l} 
Case Reports $\boldsymbol{h}$ & $\begin{array}{l}\text { Case Rep Dermatol 2010;2:55-59 } \\
\text { D01: } 10.1159 / 000313821\end{array}$ & Published online: April 27, 2010 & $\begin{array}{l}\text { @ 2010 S. Karger AG, Basel } \\
\text { ISSN 1662-6567 } \\
\text { www.karger.com/cde }\end{array}$ \\
\hline
\end{tabular}

The histopathologic study of two wrist and back lesions showed an atrophic epidermis, homogenization of the upper dermis and a mid-dermal lymphocytic infiltrate, consistent with lichen sclerosus et atrophicus (fig. 3 ).

The patient was diagnosed as LS simulating a distribution of LP, and topical corticosteroid treatment produced a clear improvement of the lesions.

\section{Conclusions}

LP and LS share several common features, including lymphocytic infiltration at the dermal-epidermal junction, clinical involvement of both skin and mucosa, erosive disease of mucosal surfaces, and the occasional occurrence of squamous cell carcinoma at the site of chronic, erosive mucosal lesions [1].

There are only rare reports of concurrent LP and LS, but the number of reported cases of LS with LP-like distribution is even lower [1].

Cases have been reported of simultaneous LS and localized scleroderma (morphea) in the same patient and even within the same lesion $[2,3]$. Different authors have suggested that both processes (LS and LP) are part of a clinical spectrum. A parallel exists between LP/LS and graft-versus-host disease (GVHD), where in early chronic GVHD, lesions appear lichenoid, and in late chronic GVHD, sclerotic lesions predominate. The pathogenesis is immunological and is considered on the spectrum of GVHD. It is therefore tempting to speculate on the immunological relationship between LP and LS [3, 4].

In LS and LP, there is a strong dermal inflammatory infiltrate comprised of $\mathrm{T}$ lymphocytes. In LS, a specific T-cell response to BP180, a structural protein of the basement membrane zone, has been demonstrated. The demonstration of the presence of circulating autoantibodies to extracellular matrix protein and to basement membrane zone (BMZ) components, chiefly BP180, suggests that autoimmunity to these components might contribute to pathogenesis $[5,6]$. This contrasts with LP and cutaneous LS which, for the moment, have not been shown to be associated with an autoimmune disorder.

In spite of clinical and histological similarities and possible common immunological origin, there are a few cases of concurrence of LS and LP, and LS simulating LP.

We report a new case of LS with a typical distribution of LP in a young woman. 
Fig. 1. Small polygonal papules, atrophic, located on the front of the wrists.

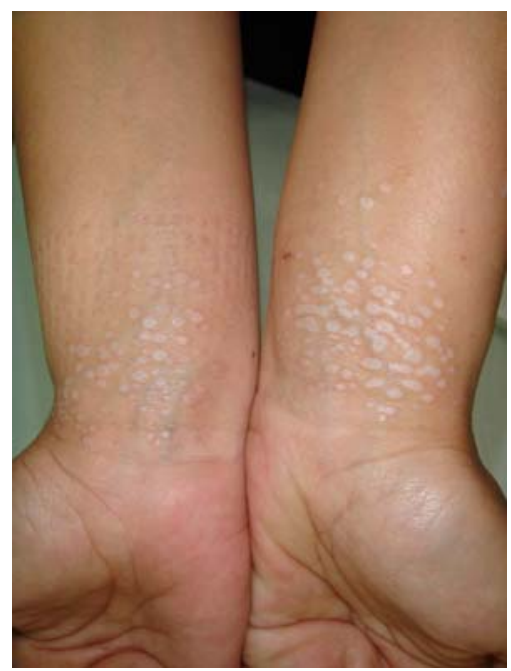

Fig. 2. Small polygonal papules, atrophic, located on the dorsum of the feet.

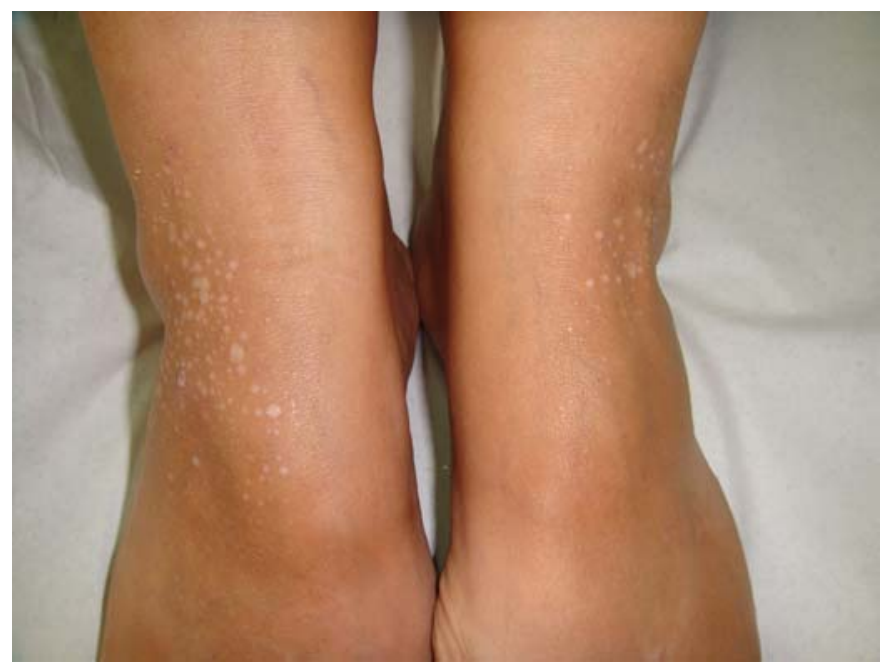


Fig. 3. Biopsy of the left wrist plaque showed a thinned epidermis with superficial dermal collagen homogenization and a mid-dermal lymphocytic infiltrate, consistent with LS. HE. $\times 100$.

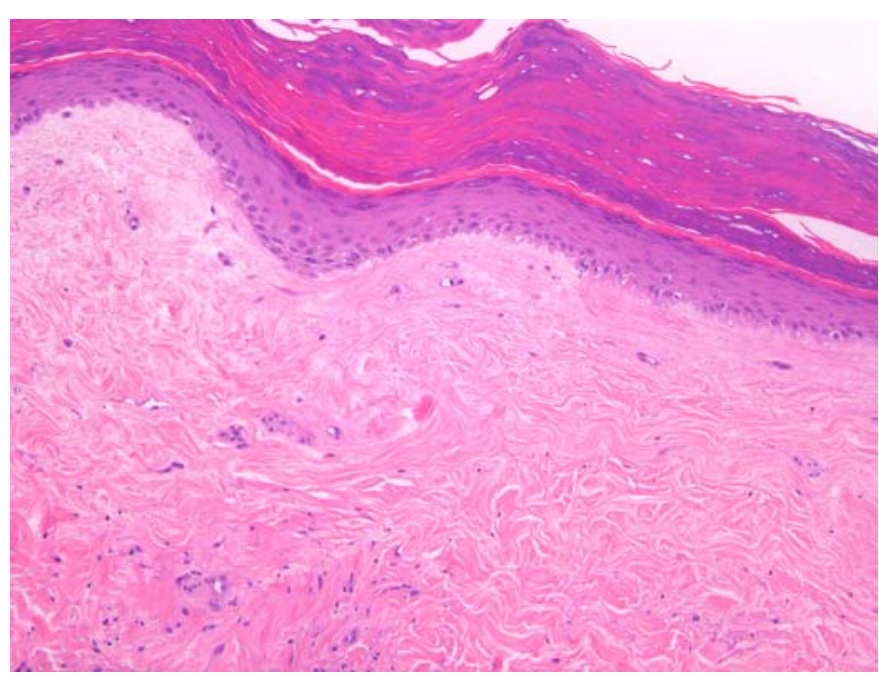




\section{References}

1 Corbalán-Vélez R, Pérez-Ferriols A: Lichen sclerosus et atrophicus affecting the wrists and left ankle and clinically simulating lichen planus. Cutis 2001;67:417419.

-2 Holmes SC, Burden AD: Lichen sclerosus and lichen planus: a spectrum of disease? Report of two cases and review of the literature. Clin Exp Dermatol 1998;23:129-131.

3 Lessani T, Gallego H, Lynch PJ: Lichen planus and lichen sclerosus overlap. Cutis 1998;61:103-104.

4 Shono S, Imura M, Ota M, et al: Lichen sclerosus et atrophicus, morphea and coexistence of both diseases. Arch Dermatol 1991;127:1352-1356.

-5 Yashar S, Han KF, Haley JC: Lichen sclerosus-lichen planus overlap in a patient with hepatitis C virus infection. Br J Dermatol 2004;150:168-169.

6 Cooper SM, Ali I, Baldo M, Wojnorowska F: The association of lichen sclerosus and erosive lichen planus of the vulva with autoimmune disease: a case-control study. Arch Dermatol 2008;144:1432-1435. 